PREPARED FOR THE U.S. DEPARTMENT OF ENERGY, UNDER CONTRACT DE-AC02-76CH03073

PPPL-3474

PPPL-3474

UC-70

Pulse Compression in Plasma: Generation of Femtosecond

Pulses Without CPA

by

G. Shvets, N.J. Fisch, A. Pukhov, and J. Meyer-ter-Vehn

July 2000

$=$ PPPL

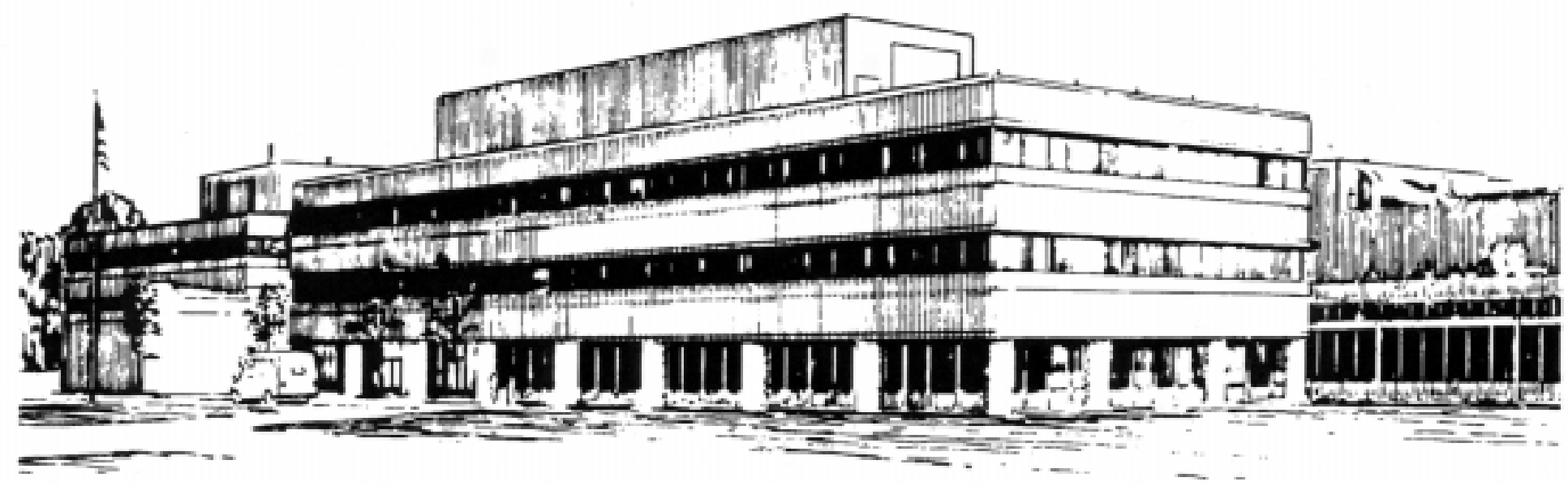

PRINCETON PLASMA PHYSICS LABORATORY PRINCETON UNIVERSITY, PRINCETON, NEW JERSEY 


\section{PPPL Reports Disclaimer}

This report was prepared as an account of work sponsored by an agency of the United States Government. Neither the United States Government nor any agency thereof, nor any of their employees, makes any warranty, express or implied, or assumes any legal liability or responsibility for the accuracy, completeness, or usefulness of any information, apparatus, product, or process disclosed, or represents that its use would not infringe privately owned rights. Reference herein to any specific commercial product, process, or service by trade name, trademark, manufacturer, or otherwise, does not necessarily constitute or imply its endorsement, recommendation, or favoring by the United States Government or any agency thereof. The views and opinions of authors expressed herein do not necessarily state or reflect those of the United States Government or any agency thereof.

\section{Availability}

This report is posted on the U.S. Department of Energy's Princeton Plasma Physics Laboratory Publications and Reports web site in Calendar Year 2000. The home page for PPPL Reports and Publications is: http://www.pppl.gov/pub_report/

DOE and DOE Contractors can obtain copies of this report from:

U.S. Department of Energy

Office of Scientific and Technical Information

DOE Technical Information Services (DTIS)

P.O. Box 62

Oak Ridge, TN 37831

Telephone: (865) 576-8401

Fax: (865) 576-5728

Email: reports@adonis.osti.gov

This report is available to the general public from:

National Technical Information Service

U.S. Department of Commerce

5285 Port Royal Road

Springfield, VA 22161

Telephone: $1-800-553-6847$ or

(703) $605-6000$

Fax: (703) 321-8547

Internet: http://www.ntis.gov/ordering.htm 


\title{
Pulse compression in plasma: generation of femtosecond pulses without CPA
}

\author{
G. Shvets and N. J. Fisch \\ Department of Astrophysical Sciences, Princeton University, Princeton, NJ 08540 \\ tel. (609)-243-2609, fax (609)-243-2662, e-mail gena@pppl.gov \\ A. Pukhov and J. Meyer-ter-Vehn \\ Max-Planck-Institut für Quantenoptik, D-85748 Garching, Germany
}

\begin{abstract}
Laser pulses can be efficiently compressed to femto- second duration when a smallerfrequency short pulse collides with high frequency long pulse in rare plasma, absorbing most of its energy. The mechanism of short pulse amplification is nonlinear superradiance. (C) 2000 Optical Society of America OCIS codes: (140.6630) Superradiance, superfluorescence; (350.5400) Plasmas
\end{abstract}

The motivation for this work is to introduce a novel method of compressing laser pulses to femtosecond duration using tenuous plasma as the nonlinear medium for parametric conversion of the energy of a long higher-frequency laser beam into the energy of a short lower-frequency laser pulse. The standard approach to generating high-intensity ultra-short laser pulses is Chirped Pulse Amplification [1] (CPA), in which a laser pulse is stretched, amplified, and re-compressed. CPA-based optical systems have been shown to generate sub-picosecond petawatt laser pulses $[2,3]$ with up to $500 \mathrm{~J}$ per pulse. The pulse energy is limited by the thermal damage to the compression gratings which become large and expensive for $\mathrm{kJ}$ pulses. Possible route to increasing peak intensity is to decrease pulse duration, which can be achieved by using broad bandwidth parametric amplifiers [4]. This does not, however, ease the energy restriction.

In this talk I introduce a novel approach to pulse compression which does not require pulse stretching/compression. We demonstrate how an ultra-short pulse can be amplified by several orders of magnitude by colliding with a long counter-propagating pumping laser in the plasma $[5,6]$. The main advantage of using the plasma medium for amplification is that there is no thermal damage threshold - fresh plasma can be used for each shot. The counter-propagating geometry is chosen for two reasons. First, it enables the short pulse to sweep through the entire pump absorbing most of its energy and integrating over all the inhomogeneities and imperfections of the pumping signal.

Second, the cold electron plasma exhibits remarkable properties as a parametric medium: its $\chi_{3}$ coefficient has a very strong dependence on the wavenumber difference $\Delta k$ between the two interacting laser pulses. In particular, for counter and co-propagating lasers ( $\uparrow \downarrow$ and $\uparrow \uparrow$, respectively) the ratio of the $\chi_{3}$ coefficients is $\chi_{3}(\uparrow \downarrow) / \chi_{3}(\uparrow \uparrow) \approx 4 \omega_{0}^{2} / \omega_{p}^{2}[7]$, where $\omega_{0}$ and $\omega_{1}$ are the frequencies of the amplified signal and the pump, $\omega_{p}=\left(4 \pi e^{2} n_{0} / m\right)^{1 / 2}$ is the plasma frequency, $n_{0},-e$, and $m$ are the plasma density, electron charge, and mass, respectively. In a tenuos plasma this ratio can be several hundred. This distinguishes parametric amplification in cold electron plasma from that in gasses, liquids, and fibers. Therefore, a short pulse can be rapidly amplified by backscattering a counter-propagating pump without suffering from forward-propagating instabilities (such as Raman forward scattering and filamentation) [6]. $\chi_{3}$ of the electron plasma can be understood as follows: electron density perturbation is ponderomotively driven by the periodic intensity pattern produced by the interference between the pumping beam (PB) and the amplified beam (AB). This density perturbation then serves as a grating which scatters the pump.

We suggest two regimes of pulse compression in plasma. In the first (Compton) regime a finite-amplitude ultra-short pulse of duration $\tau_{L} \sim 2 \omega_{p}^{-1}$ is injected. Accessing this regime requires a sufficiently intense initial pulse: $4 \omega_{0}^{2} a_{0} a_{1} \geq \omega_{p}^{2}$, where $a_{0,1}=e A_{0,1} / m c^{2}$ are the normalized vector potentials of the $\mathrm{AB}$ and $\mathrm{PB}$, respectively. When this condition is satisfied, electron motion is determined by the ponderomotive force and not by the space-charge electric field of the plasma wave. The resonance condition for the plasma wave excitation $\Delta \omega=-\omega_{p}$ doesn't need to be satisfied precisely, making the amplification process robust to plasma inhomogeneity.

Numerical simulation of the pulse amplification in this regime using particle-in-cell (PIC) numerical code is shown in Fig. 1, where the amplification of a short (initially $\tau_{L}=10 \mathrm{fs}$ ) $\lambda_{0}=1 \mu \mathrm{m}$ laser pulse is demonstrated 


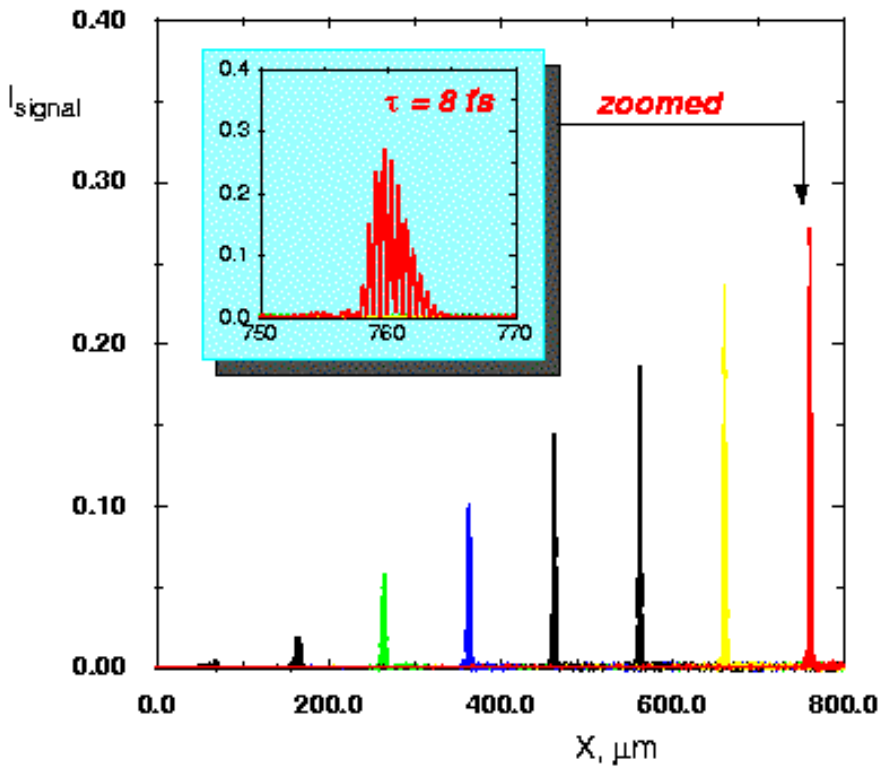

Fig. 1. Amplification of $10 \mathrm{fs}$ pulse with initial intensity $I_{0}=3.5 \times 10^{15} \mathrm{~W} / \mathrm{cm}^{2}$ by a counter-propagating pump with the same intensity $I_{1}=I_{0}$ inside the plasma with density $n_{0}=1.5 \times 10^{19} \mathrm{~cm}^{-3}$. After 700 microns the signal intensity grows by factor 60 .

as it propagates through $700 \mu \mathrm{m}$ of the plasma while interacting with a long counter-propagating pulse whose frequency is 3\% higher. Simulation parameters are given in the caption. As Fig. 1 indicates, the short pulse intensity is amplified by a factor 60 while its duration shrinks to about 8 fs (see the inset). We demonstrated numerically that the efficiency of energy conversion from the long to short pulse can be quite high. In Fig. 2 a collision between a somewhat stronger short pulse with $a_{0}=0.07\left(I_{0}=1.3 \times 10^{16} \mathrm{~W} / \mathrm{cm}^{2}\right)$ and the pump with $a_{1}=0.025\left(I_{0}=1.7 \times 10^{15} \mathrm{~W} / \mathrm{cm}^{2}\right)$ is demonstrated. As Fig. 2(b) indicates, the pump is $40 \%$ depleted by the short pulse.

Superradiant amplification is a strongly nonlinear process. The dependence of the short pulse intensity and duration on the propagation distance $z$ can be qualitatively estimated as

$$
\left|a_{0}\right|^{2} \sim \frac{\left|a_{1}\right|^{2} \omega_{p}^{4} z^{2}}{16 \omega_{0}^{2} c^{2}} \quad \tau_{L} \approx \frac{2}{\omega_{p} a_{1}}\left(\frac{c}{\omega_{0} z}\right)^{1 / 2}
$$
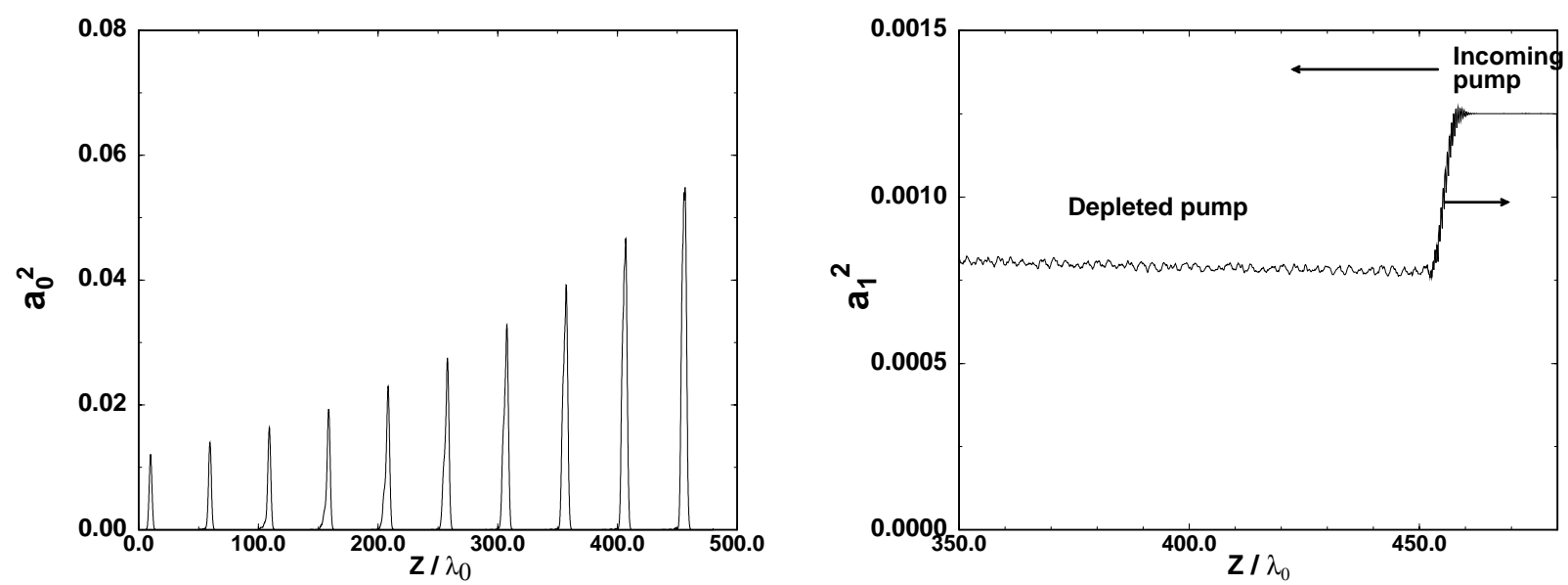

Fig. 2. Large pump depletion regime modeled by PIC simulation. Pump $a_{1}=0.025$, initial signal $a_{0}=0.07$ and $\tau_{L}=1 / \omega_{p}$, plasma $n_{0}=10^{19} \mathrm{~cm}^{-3}$. Left figure: short pulse amplification (series of snapshots); right figure: $40 \%$ pump depletion by the short pulse. 

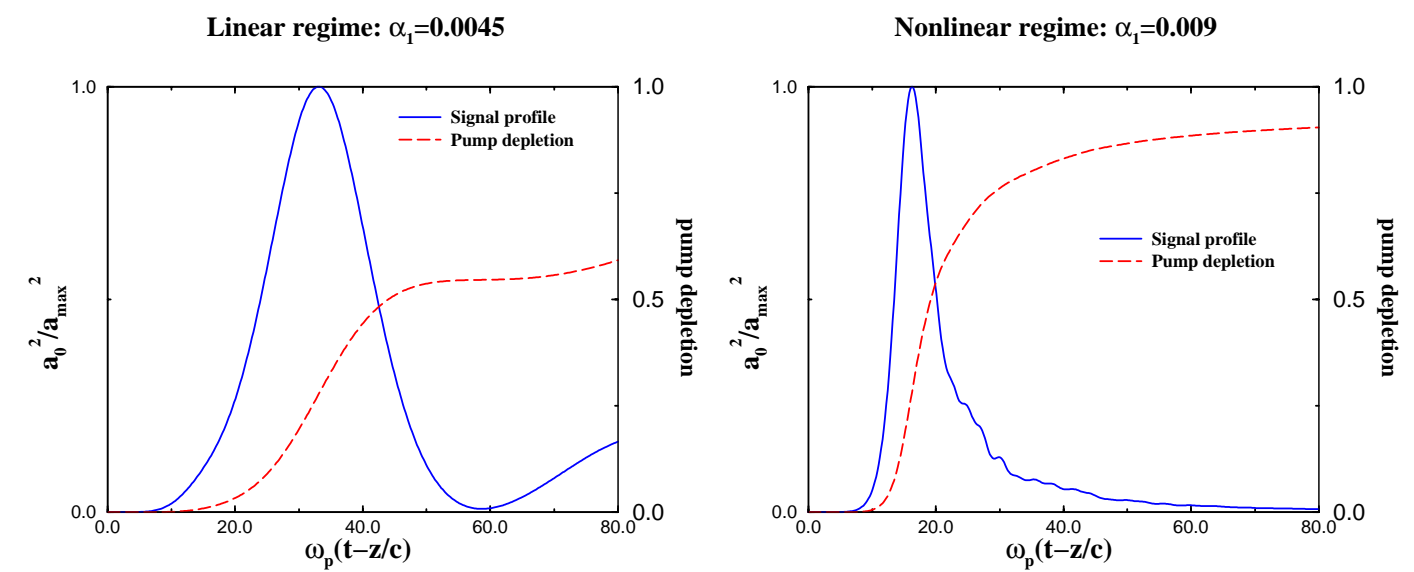

Fig. 3. Normalized intensity profiles for the amplified pulse for two pump amplitudes: below (left) and slightly above (right) the wavebreaking amplitude $a_{\mathrm{wb}}=0.008$. In this example $n_{0}=10^{19} \mathrm{~cm}^{-3}$ and the amplification distance $L=4 \mathrm{~mm}$.

Quadratic dependence of the short pulse intensity on the propagation distance (and, therefore, on the number of electrons $\mathcal{N}$ encountered by the pulse) gives this amplification process its name [8]. The dependence of $\tau_{L}$ has a somewhat unusual scaling $1 / \sqrt{\mathcal{N}}$. This is because the pulse narrowing is caused by the laser recoil and not by the pump depletion. In particular, the characteristic bounce frequency of a plasma electron inside the ponderomotive potential created by the interference of the pump and the pulse is given by $\omega_{B}=2 \omega_{0} \sqrt{a_{0} a_{1}}$. In the Compton regime frequency replaces the Rabi frequency which, in atomic systems, scales $\sim a_{0}$. We've demonstrated [9] that in the absense of significant pump depletion pulse amplification is described by the self-similar solution which maintains $\omega_{B} \tau_{L} \approx \pi$ throughout the amplification process.

Unlike the Compton regime, where the dominant nonlinear mechanism is particle trapping by the ponderomotive potential, in the Raman regime the motion of the plasma electrons remains linear, and the dominant nonlinearity is the pump depletion. This regime is described by the standard set of equations for three-wave interaction. Frequency detuning between the pump and the pulse has to be precisely equal to the plasma frequency: $\omega_{1}-\omega_{0}=\omega_{p}$, imposing stringent requirements on plasma homogeneity. The important figure of merit that separates the two regimes is the ratio of the pump amplitude $a_{1}$ to the critical $a_{\mathrm{wb}}=0.25\left(\omega_{p} / \omega_{0}\right)^{3 / 2}$ : for $a_{1}>a_{\mathrm{wb}}$ plasma wave breaks before the pump is depleted, and Compton regime is accessed. In the opposite low-intensity limit of $a_{1}<a_{\mathrm{wb}}$ three-wave (Raman) description is appropriate.

Raman regime is characterized by the "Burnham-Chiao ringing" [10]: periodic energy exchange between the pulse and the pump due to the long-lived coherent plasma wave. We demonstrated numerically that this ringing can be suppressed by operating near the wavebreaking limit $a_{1} \approx a_{\mathrm{wb}}$. In this case the plasma wave breaks and thermalizes near the intensity maximum of the pulse. The suppression of the ringing in this regime is demonstrated in Fig. 3: only $50 \%$ of the pump energy flows into the leading spike in the linear case, and about $80 \%$ in the nonlinear case.

\section{References}

1. D. Strickland and G. Mourou, Opt. Commun. 56, 219 (1985).

2. G. A. Mourou, C. P. J. Barty, and M. D. Perry, Phys. Today, p. 22, January 1998.

3. M. H. Key et. al., Phys. Plasmas 5, 1966 (1998).

4. I. N. Ross et. al., Opt. Comm. 144, 125 (1997).

5. G. Shvets, N. J. Fisch, A. Pukhov, and J. Meyer-ter-Vehn, Phys. Rev. Lett. 81, 4879 (1998).

6. V. M. Malkin, G. Shvets, and N. J. Fisch, Phys. Rev. Lett. 82, 4448 (1999).

7. G. Shvets and A. Pukhov, Phys. Rev. E 59, 1033 (1999).

8. R. Dicke, Phys. Rev. 93, 99 (1954).

9. G. Shvets, N. J. Fisch, A. Pukhov, and J. Meyer-ter-Vehn, in preparation (2000).

10. D. C. Burnham and R. Y. Chiao, Phys. Rev. 188, 667 (1969). 
The Princeton Plasma Physics Laboratory is operated by Princeton University under contract with the U.S. Department of Energy.

\author{
Information Services \\ Princeton Plasma Physics Laboratory \\ P.O. Box 451 \\ Princeton, NJ 08543
}

Phone: 609-243-2750

Fax: 609-243-2751

e-mail: pppl_info@pppl.gov

Internet Address: http://www.pppl.gov 